\title{
Influência do ângulo de incidência nos ganhos de calor solar através de materiais transparentes
}

\author{
The influence of the incidence angle on the heat gains \\ through transparent materials
}

\section{J oaquim Pizzutti dos Santos Maurício Roriz}

J oaquim Pizzutti dos Santos Departamento de Estruturas e Construção Civil, Centro de Tecnologia

Universidade Federal de Santa Maria Faixa de Camobi, km 09, Campus Universitário Santa Maria - RS - Brasil CEP 97119-900

Tel.: (55) 220-914 Ramal 9144 E-mail: joaquimpizzutti@hotmail.com

Maurício Roriz Departamento de Engenharia Civil, Centro de Ciênias Exatas e de Tecnologia Universidade Federal de São Carlos Via Washington Luís, Km 235 Caixa Postal 67

São Carlos - SP - Brasi CEP 13565-905

Tel.: (16) 3351-8262 E-mail: m.roriz@terra.com.br

Recebido em 10/10/11

Aceito em 01/02/12

\section{Resumo}

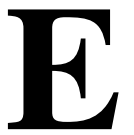

ste trabalho apresenta uma equação genérica que relaciona a variação do Fator Solar com o ângulo de incidência. As curvas resultantes desta variação, para vidros e outros materiais transparentes, foram obtidas a partir de experimentos de espectrofotometria para reflexão e transmissão, realizados para diferentes ângulos de incidência. Desta forma, foram identificados grupos de materiais com comportamento semelhante de variação do Fator Solar em função do ângulo. Para cada um dos grupos foi definido um material de referência, com valores de Fator Solar representando o comportamento médio do grupo. Para estes materiais foram obtidas equações gerais da curva de variação do Fator Solar, que permite calcular o ganho de calor solar total considerando a variação do ângulo de incidência da radiação ao longo de um período de tempo. $\mathrm{O}$ valor desses ganhos para qualquer material do mesmo grupo pode ser obtido aplicando um coeficiente de correção, que é o valor do Fator Solar para a incidência normal do material considerado. Os valores para esse coeficiente são propostos neste trabalho para um grande número de materiais ou podem ser obtidos a partir de catálogos.

Palavras-chaves: Materiais transparentes. Fator Solar. Ganhos de calor solar.

\section{Abstract}

This paper presents a generic equation which relates the Solar Factor variation with the incidence angle. The curves that result from this variation, for glasses and other transparent materials, were obtained through spectrophotometric experiments for transmission and reflection at different incidence angles. Groups of materials with similar variation behaviors according to the angle were identified. A reference material was defined for each group, representing the average behavior of the group. For these materials general equations of the Solar Factor variation curve were obtained, which allowed the calculation of the total heat gain, considering the change in the angle of radiation incidence over a period of time. The value of these gains for any material from the same group can be obtained by applying a correction coefficient, which is the Solar Factor value for the normal incidence of the material. In this study, those values are proposed for a large number of materials, or can be obtained from catalogs.

Keywords: Transparent materials. Solar factor. Solar Heat Gain. 


\section{Introdução}

O progressivo e diversificado uso de elementos transparentes nas vedações das edificações e a busca por materiais energeticamente mais eficientes levaram ao desenvolvimento de materiais mais apropriados a usos específicos, o que acarretou uma ampla variação de suas características, aumentando significativamente o número de opções disponíveis aos projetistas. Esse fato dificulta o processo de especificação desses materiais, aumentando o número de variáveis a ser analisadas, que envolvem considerações de estética, de conforto visual, térmico e acústico, além de segurança, vista externa, custos e outros. Cledwyn-Davies (1993) já associava o grande avanço na tecnologia de produção dos vidros float e seus tratamentos à demanda cada vez maior, por parte dos projetistas, de produtos com boa aparência, controle solar e economia de energia.

O cálculo dos ganhos de calor através de um material transparente envolve basicamente três variáveis:

(a) a intensidade da radiação solar incidente;

(b) o ângulo de incidência da radiação; e

(c) o fator solar (FS) do elemento transparente para o ângulo de incidência no instante considerado.

Fazendo-se o somatório desses valores ao longo de um espaço de tempo qualquer, obtém-se o ganho total no período considerado.

Nos diversos programas computacionais hoje disponíveis que realizam o cálculo de carga térmica através desses materiais, os dados referentes às características ópticas dos diversos elementos transparentes encontram-se nos bancos de dados do programa e nem sempre possuem o mesmo detalhamento e precisão que o processo computacional envolvido, gerando resultados distorcidos.

A consideração do real ângulo de incidência da radiação torna esse cálculo mais preciso se comparado com a utilização de dados para incidência normal da radiação, principalmente quando envolve ângulos acima de 50o, devido às variações expressivas das características ópticas dos materiais transparentes a partir desse ângulo (PFROMMER et al., 1995). Nesse sentido, Caram (1998) comenta a questão da falta de dados mais confiáveis e completos nos catálogos dos fabricantes de vidros. Esses catálogos fornecem valores do fator solar de seus produtos para condições climáticas e de posição do sol padronizadas, sendo estabelecido o sol a 30o acima do horizonte, incidindo perpendicularmente ao plano de incidência (incidência normal), com as temperaturas externas e internas iguais (COMPANHIA..., 1993). Os valores assim obtidos podem ser adotados como valores iniciais, como uma referência de comparação, ou para cálculos em que as condições são próximas dessas, pois em geral as condições reais de utilização são bastante diferenciadas, necessitando um refinamento maior na obtenção desse fator.

Marinoski (2010) desenvolveu um calorímetro para medição do FS de vidros e janelas completas, com ou sem elementos de proteção solar, sob condições reais de utilização. O dispositivo emprega dois sistemas diferentes para quantificar o fluxo de calor que penetra através do elemento de fechamento testado, obtendo resultados bastante coerentes para o valor do FS, mas tendo limitação na variação do ângulo de incidência, não possibilitando a medição do FS para qualquer ângulo de incidência.

Da mesma maneira, trabalhos de pesquisa que envolvem cálculo de FS para vidros translúcidos (SANTOS; FERNANDES, 2008) ou policarbonatos translúcidos (SANTOS; FERNANDES, 2009) também têm a limitação de não ser possível a medição da transmitância e da refletância desses materiais a ângulos variados, pois a obtenção desses valores envolve medições de transmissão e de reflexão através de espectrofotômetro, com o uso de esfera integradora, 0 qual somente permite 0 posicionamento da amostra para a incidência normal do feixe de luz nela.

A possibilidade demonstrada neste trabalho, da obtenção do FS para diferentes ângulos a partir do valor para incidência normal, reduz o número de ensaios e a necessidade de ensaios mais elaborados a ângulos variados, os quais possuem um maior grau de dificuldade.

A maneira mais prática de consideração da variação do valor do FS com o ângulo de incidência no cálculo de ganhos de calor é a determinação do FS para os diferentes ângulos para um material de referência, com a obtenção do FS dos demais materiais apenas para incidência normal. Assim, os cálculos dos ganhos de calor são realizados para o material de referência, sendo, após, efetuado um ajuste para outro elemento qualquer a ser utilizado. Esse ajuste é feito através da multiplicação do valor obtido do cálculo inicial por um coeficiente resultante da relação entre o FS do elemento qualquer e o FS do material de referência, ambos para incidência normal, denominado usualmente de coeficiente de 
sombreamento, quando o material de referência é o vidro incolor de $3 \mathrm{~mm}$. Esse coeficiente é definido como a relação entre o ganho total de radiação solar, tanto por transmissão como por absorção e reemissão, de uma abertura externa ou combinação de abertura e proteção solar qualquer, sob determinado conjunto de condições ambientais e de incidência da radiação solar, e uma abertura de referência composta de vidro simples de $3 \mathrm{~mm}$ incolor, sob as mesmas condições. Para facilitar o cálculo dos ganhos solares, a ASHRAE (AMERICAN..., 1997) fornece tabela com o coeficiente de sombreamento de alguns tipos de aberturas utilizadas e também a intensidade da radiação e o ganho de calor solar do vidro de referência, para diferentes orientações de fachada e latitudes, levando em consideração o ângulo de incidência solar.

A utilização de um material de referência para o cálculo possui vantagens por possibilitar o uso de tabelas para cálculos, ou por simplificar as rotinas no cálculo computacional. Além disso, o número de ensaios é reduzido, eliminando a necessidade de ensaios a ângulos variados para a grande quantidade de materiais transparentes existentes.

No entanto, esse processo somente proporciona uma precisão satisfatória se os elementos correlacionados apresentarem comportamentos semelhantes de variação do FS com o ângulo de incidência, pois o valor do fator de correção deve ser único, devendo manter-se constante, independentemente do ângulo. O material adotado como referência, atualmente, para todos os demais tipos de materiais transparentes é o vidro incolor de $3 \mathrm{~mm}$. Esse fato gera distorções devido às diferenças de variação do FS com o ângulo de incidência desse vidro em relação a diversos outros tipos de materiais transparentes, mais evidentes em ângulos superiores a 50o.

Este trabalho tem como objetivo desenvolver um procedimento para facilitar a consideração do ângulo de incidência da radiação solar no cálculo de ganhos de calor através de elementos transparentes, determinando materiais de referência mais apropriados, definidos a partir da observação, dentro do conjunto de materiais transparentes disponíveis no mercado, de grupos de materiais com comportamentos similares de variação do fator solar com o ângulo de incidência. A partir das curvas de variação do FS dos materiais de referência, que expressam a variação média dos materiais de cada grupo, elaborou-se uma equação genérica de variação do FS em função do ângulo de incidência, com parâmetros específicos para cada grupo. Os dados calculados a partir dessa fórmula servem como base de cálculo de ganhos de calor para esses materiais, devendo ser posteriormente corrigidos para um material qualquer do grupo a partir do FS para incidência normal do material desejado.

Procedimento semelhante foi utilizado em trabalho realizado por Santos e Roriz (2007), no qual foi levado em consideração o comportamento de variação da transmitância da luz visível (Tv) dos materiais transparentes, resultando em equações genéricas de variação da Tv em função do ângulo de incidência, que podem ser utilizadas em cálculos de admissão de luz natural em ambientes ao longo de um período.

\section{Metodologia}

O desenvolvimento deste trabalho inicia-se com o cálculo do fator solar dos diferentes tipos de materiais transparentes para ângulos variando de 0 a 90 graus. A partir desses valores os materiais são reunidos em grupos com comportamento semelhante de variação do FS com o ângulo, sendo então definido, para cada grupo, um material de referência, para o qual é estabelecida uma equação genérica que expresse a variação do FS em função do ângulo de incidência.

\section{Cálculo do fator solar para diferentes ângulos de incidência}

O fator solar para os diferentes ângulos de incidência foi calculado como $\mathrm{FS}=\mathrm{T}+\mathrm{Ni}$. A, sendo $\mathrm{T}$ a transmitância, Ni a parcela de energia absorvida que vai para o interior da edificação, e A a absortância, calculada por $A=100-(T+R)$, onde $R$ é a refletância.

Para a determinação das transmitâncias e refletâncias foram realizados ensaios espectrofotométricos a ângulos de incidência variados para 60 tipos de materiais transparentes, reunindo os vidros comuns, laminados comuns, laminados refletivos, refletivos pirolíticos, refletivos a vácuo, policarbonatos e acrílicos (SANTOS, 2002).

As medidas de reflexão foram realizadas com a utilização do espectrofotômetro Cary 17/Olis. Para a obtenção de dados de reflexão através desse equipamento foi necessária a montagem de um dispositivo que desviasse o feixe de luz, para que a incidência sobre a amostra fosse em ângulos variados e para que o feixe medido, após incidir sobre a amostra, fosse o feixe refletido, e não o transmitido (SANTOS, 2002).

As medidas de transmissão para ângulos variados de incidência foram efetuadas da mesma forma que a reflexão, sendo empregado para essa finalidade o espectrofotômetro Cary 5G. Este 
aparelho possui o Ângulo de Brewster, dispositivo apropriado para variação no ângulo de posicionamento da amostra em relação ao feixe incidente.

Tanto na reflexão quanto na transmissão o ângulo variou de 0 a 90 graus, com intervalo de $10^{\circ}$, e o espectro utilizado nas medições foi de 300 a 2.000 $\mathrm{nm}$, com intervalo de medições de $4 \mathrm{~nm}$.

A curva espectrofotométrica resultante do ensaio foi ponderada em relação ao espectro solar padrão utilizado, sendo multiplicada pelo montante de energia solar que incide em cada comprimento de onda, conforme a ASTM-E892 (AMERICAN..., 1997). A transmitância e a refletância foram calculadas a partir da relação entre a integral das curvas de transmissão e de reflexão ponderadas e a integral da curva de radiação solar incidente, ou seja, entre o total de energia que o material reflete ou transmite e o total de energia solar que chega.

No cálculo do FS foi considerada uma velocidade do vento de 0 a $3 \mathrm{~m} / \mathrm{s}$ e a superfície na posição vertical ou horizontal, pois esses parâmetros influem na parcela da energia absorvida que migra para o interior da edificação, calculada conforme Santos (2002).

\section{Definição dos grupos de materiais}

Para a visualização e a determinação de grupos de materiais com comportamento semelhante de variação do FS com o ângulo de incidência é utilizada a análise comparativa da curva de variação do valor do FS relativo (FSrel), cujos valores resultam da divisão do FS de cada ângulo pelo FS para incidência normal.

A Figura 1 apresenta um exemplo de análise comparativa do FSRel para os vidros comuns.
Observa-se nessa figura o comportamento similar de variação desses tipos de vidros, o que leva à identificação de que esses materiais podem ser considerados no mesmo grupo para essa finalidade.

Na metodologia de cálculo e definição das curvas de variação do FS foi considerada uma velocidade do vento de $2 \mathrm{~m} / \mathrm{s}$ e a superfície na posição vertical; no entanto, foram também analisadas as tabelas de FS para velocidades de 0 a $3 \mathrm{~m} / \mathrm{s}$, variando de 1 em $1 \mathrm{~m} / \mathrm{s}$, e vedações na posição horizontal, utilizando-se o mesmo procedimento adotado nas tabelas para a velocidade do vento de $2 \mathrm{~m} / \mathrm{s}$. Observou-se, nessa análise, que a consideração de diferentes velocidades do vento e da orientações da superfície transparente não acarreta, para nenhum dos grupos de elementos determinados, uma alteração sensível do modo de variação do FSrel com o ângulo de incidência.

\section{Definição dos materiais de referência dos grupos}

A observação de grupos de materiais com comportamentos semelhantes permitiu a definição de um material de referência para cada grupo, que expressasse o comportamento médio dele. Foram adotados materiais hipotéticos de referência, com valores de FS obtidos a partir dos valores médios para cada ângulo do FSrel de todos os materiais do grupo. Esses materiais terão a curva de variação do FS com valores partindo de 1, para incidência normal, e terminando em 0 , para $90^{\circ}$. A proposição de adoção desses materiais é o objetivo principal deste trabalho, pois eles vão servir de base para o cálculo dos ganhos de calor ao longo de um período.

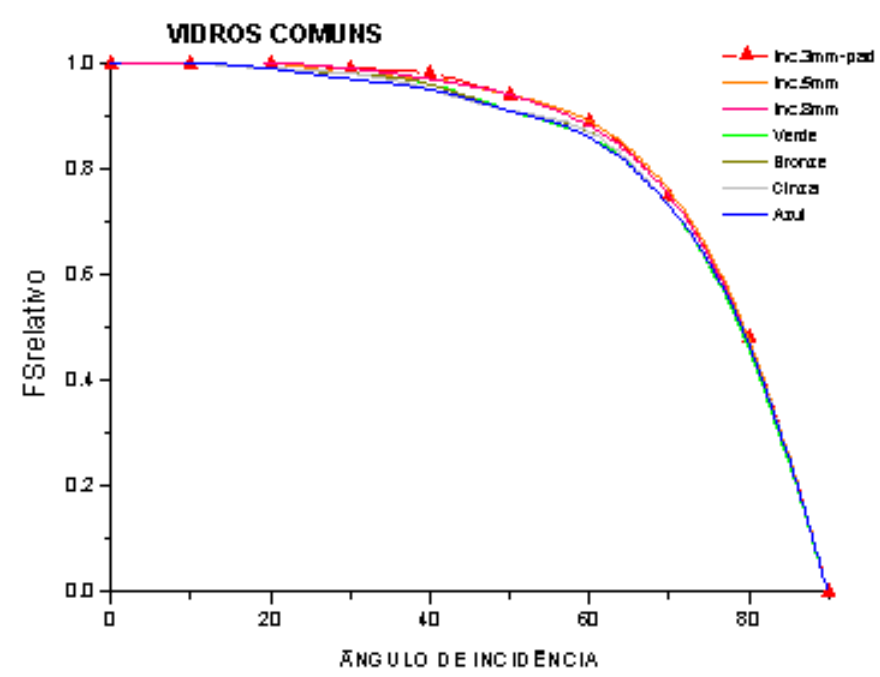

Figura 1 - Variação do FSrel com o ângulo de incidência: vidros comuns 
Um exemplo do processo de definição desses materiais, para os materiais do Grupo 1, está apresentado na Tabela 1.

A utilização desses materiais hipotéticos, que reproduzem para cada ângulo o padrão médio de variação do FS dos materiais de cada grupo, apresenta as seguintes vantagens adicionais em relação à utilização de um material de referência existente no grupo:

(d) maior precisão como padrão de variação do FS ao longo dos ângulos, por apresentarem o comportamento médio de variação dos diversos materiais componentes do grupo;

(e) para a obtenção da curva de variação de um material qualquer do grupo considerado, todos os valores da curva de referência são corrigidos igualmente a partir da multiplicação por um coeficiente, que é obtido pela divisão do FS de um material qualquer pelo FS do material de referência, ambos para incidência normal. Como o valor do FS para incidência normal do material de referência é igual a 1, o valor do coeficiente de correção vai ser sempre igual ao valor do FS para incidência normal do material qualquer. Esse recurso possibilita o cálculo do FS para qualquer ângulo e para qualquer material do qual se conheça apenas o FS para incidência normal e o grupo de materiais a que pertença; e

(f) no caso de diferentes velocidades do vento e superfícies em posições horizontais, pode-se utilizar o mesmo material de referência definido para a velocidade de $2 \mathrm{~m} / \mathrm{s}$, usando-se, nestes casos, o coeficiente de correção apropriado para as diferentes velocidades do vento e posições de fachada, que equivale ao FS para incidência normal calculado para essas condições.

\section{Análise dos resultados}

As Figuras 2 a 5 apresentam os gráficos da variação do fator solar relativo (FSrel) em função do ângulo de incidência, fazendo-se uma comparação entre os diferentes tipos de materiais transparentes ensaiados e entre eles e o vidro incolor de $3 \mathrm{~mm}$ (material usual de referência). Foram considerados nesses gráficos ângulos entre 30 e 80 graus, pois em ângulos menores a variação e as diferenças do FS não são significativas.

Tabela 1 - Valores de FSrel e FSrel médio para os materiais do Grupo 1

\begin{tabular}{|c|c|c|c|c|c|c|c|c|c|c|c|c|}
\hline \multirow{4}{*}{ ÂNGULO } & \multicolumn{11}{|c|}{ FATOR SOLAR Relativo - GRUPO 1} & \\
\hline & \multicolumn{7}{|c|}{$\begin{array}{l}\text { VIDROS COMUNS } \\
\end{array}$} & \multicolumn{4}{|c|}{ REFLETIVOS PIROLÍTICOS } & \\
\hline & Incolor & Incolor & Incolor & Verde & Cinza & Bronze & Azul & Verde & Bronze & Cinza & Prata & \\
\hline & $3 \mathbf{~ m m}$ & $5 \mathrm{~mm}$ & $8 \mathrm{~mm}$ & $5 \mathrm{~mm}$ & $5 \mathrm{~mm}$ & $5 \mathrm{~mm}$ & $5 \mathrm{~mm}$ & $6 \mathrm{~mm}$ & $6 \mathrm{~mm}$ & $6 \mathrm{~mm}$ & $4 \mathrm{~mm}$ & \\
\hline 0 & 1,00 & 1,00 & 1,00 & 1,00 & 1,00 & 1,00 & 1,00 & 1,00 & 1,00 & 1,00 & 1,00 & \\
\hline 10 & 1,00 & 1,00 & 1,00 & 1,00 & 1,00 & 1,00 & 1,00 & 1,00 & 1,00 & 1,00 & 1,00 & \\
\hline 20 & 1,00 & 1,00 & 1,00 & 0,99 & 0,99 & 0,99 & 0,99 & 1,00 & 0,99 & 0,99 & 1,00 & \\
\hline 30 & 0,99 & 0,98 & 0,99 & 0,98 & 0,98 & 0,98 & 0,97 & 1,00 & 0,97 & 0,97 & 0,99 & \\
\hline 40 & 0,98 & 0,97 & 0,97 & 0,96 & 0,95 & 0,96 & 0,95 & 0,95 & 0,95 & 0,94 & 0,97 & \\
\hline 50 & 0,94 & 0,94 & 0,94 & 0,91 & 0,91 & 0,91 & 0,91 & 0,91 & 0,91 & 0,89 & 0,94 & \\
\hline 60 & 0,89 & 0,89 & 0,88 & 0,86 & 0,87 & 0,87 & 0,86 & 0,84 & 0,86 & 0,84 & 0,90 & \\
\hline 70 & 0,75 & 0,76 & 0,75 & 0,73 & 0,73 & 0,73 & 0,73 & 0,72 & 0,74 & 0,73 & 0,77 & \\
\hline 80 & 0,48 & 0,48 & 0,47 & 0,46 & 0,47 & \begin{tabular}{l|l}
0,47 \\
\end{tabular} & 0,47 & 0,45 & 0,46 & 0,46 & 0,48 & \\
\hline \multirow{3}{*}{ ÂNGULO } & \multicolumn{5}{|c|}{ PELÍCULAS COMUNS } & \multicolumn{6}{|c|}{\begin{tabular}{|l|} 
POLICARBONATOS E ACRÍLICO \\
\end{tabular}} & \multirow{3}{*}{$\begin{array}{c}\text { FSRel } \\
\text { MÉDIO } \\
\text { Grupo } 1\end{array}$} \\
\hline & \multirow{2}{*}{ Fumê } & \multirow{2}{*}{ Museu } & \multirow{2}{*}{ Violeta } & \multirow{2}{*}{ Bronze } & \multirow{2}{*}{ Verde } & Incolor & Cinza & Bronze & Verde & Azul & Acrílico & \\
\hline & & & & & & $5 \mathrm{~mm}$ & $5 \mathrm{~mm}$ & $5 \mathrm{~mm}$ & $5 \mathrm{~mm}$ & $5 \mathrm{~mm}$ & $5 \mathrm{~mm}$ & \\
\hline 0 & 1,00 & 1,00 & 1,00 & 1,00 & 1,00 & 1,00 & 1,00 & 1,00 & 1,00 & 1,00 & 1,00 & 1,00 \\
\hline 10 & 1,00 & 1,00 & 1,00 & 1,00 & 1,00 & 1,00 & 1,00 & 1,00 & 1,00 & 1,00 & 1,00 & 1,00 \\
\hline 20 & 1,00 & 1,00 & 1,00 & 1,00 & 1,00 & 1,00 & 0,99 & 1,00 & 1,00 & 1,00 & 0,99 & 0,99 \\
\hline 30 & 0,99 & 1,00 & 0,99 & 0,99 & 0,99 & 0,99 & 0,98 & 0,99 & 0,99 & 0,99 & 0,99 & 0,98 \\
\hline 40 & 0,96 & 0,98 & 0,97 & 0,97 & 0,97 & 0,97 & 0,96 & 0,96 & 0,97 & 0,97 & 0,97 & 0,96 \\
\hline 50 & 0,94 & 0,94 & 0,94 & 0,94 & 0,95 & 0,93 & 0,92 & 0,92 & 0,93 & 0,93 & 0,93 & 0,93 \\
\hline 60 & 0,89 & 0,88 & 0,89 & 0,88 & 0,92 & 0,87 & 0,86 & 0,86 & 0,87 & 0,88 & 0,86 & 0,87 \\
\hline 70 & 0,74 & 0,74 & 0,76 & 0,75 & 0,77 & 0,73 & 0,74 & 0,73 & 0,74 & 0,73 & 0,73 & 0,74 \\
\hline 80 & 0,48 & 0,49 & 0,50 & 0,49 & 0,51 & 0,48 & 0,48 & 0,48 & 0,48 & 0,48 & 0,47 & 0,48 \\
\hline
\end{tabular}




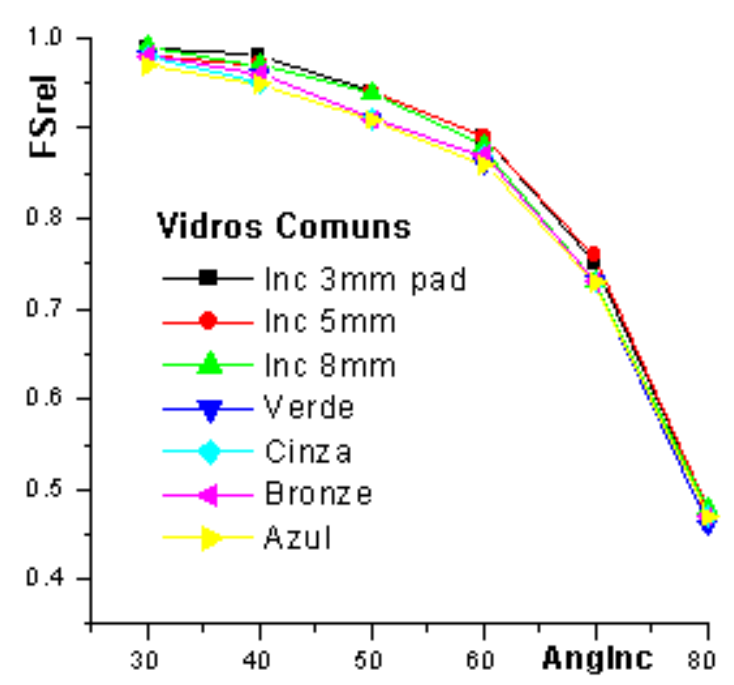

Figura 2 - Vidros comuns - FSrel em função do ângulo de incidência (Anglnc)
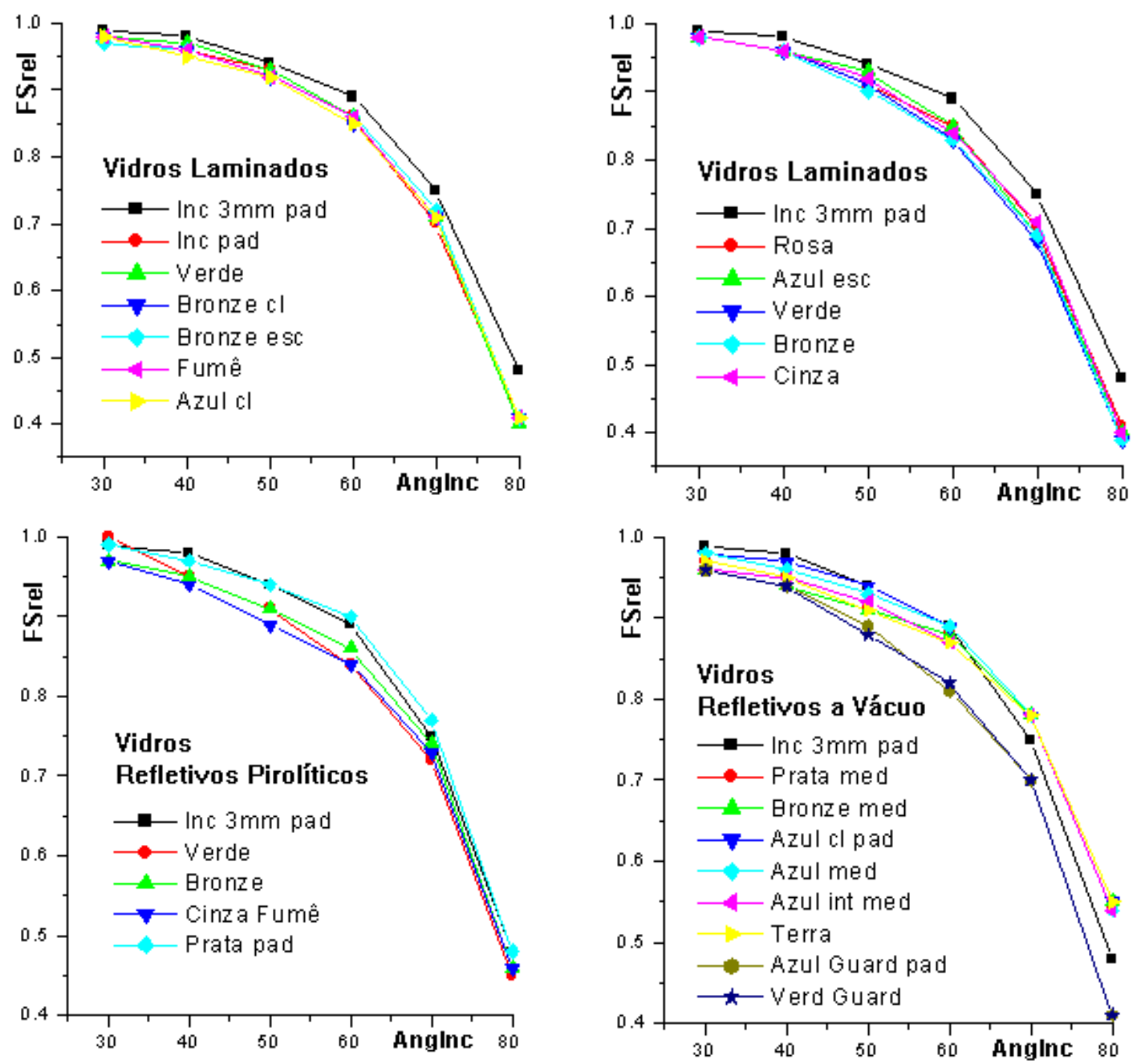

Figura 3 - Vidros laminados e vidros refletivos - FSrel em função do ângulo de incidência (Anglnc) 

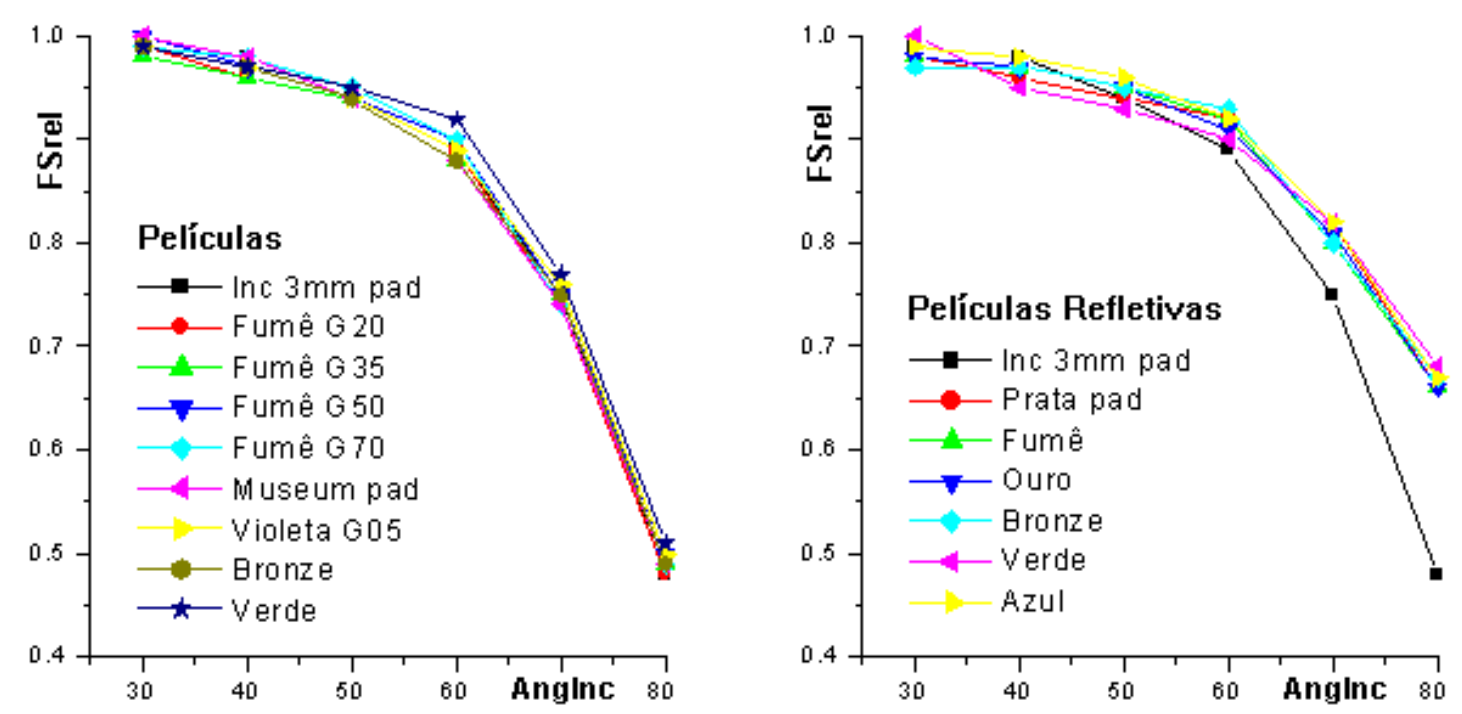

Figura 4 - Películas comuns e películas refletivas - FSrel em função do ângulo de incidência (Anglnc)

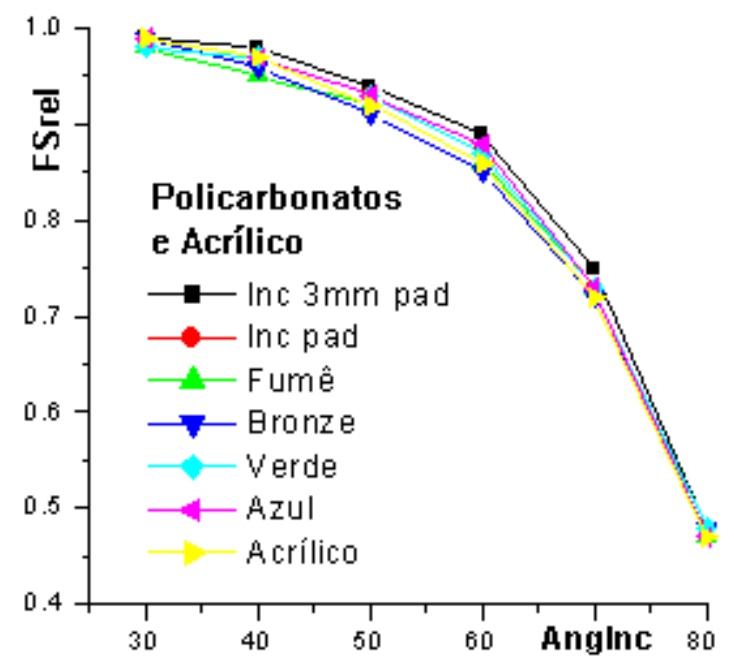

Figura 5 - Policarbonatos e acrílico - FSrel em função do ângulo de incidência (Anglnc)

A análise desses gráficos permite observar quais grupos de materiais possuem comportamento semelhante de variação do FS e podem ter um mesmo material referencial para cálculo de ganhos de calor. Dessa maneira, foi possível concluir os seguintes comportamentos dos diferentes tipos de elementos transparentes analisados:

(a) na Figura 2 observa-se que os vidros comuns mantêm entre eles uma grande semelhança de variação do FS;

(b) nos valores do FS dos vidros laminados (Figura 3) a influência da película de butiral interna acarreta, para ângulo de $80^{\circ}$, uma diminuição brusca do FS, identificando um comportamento diferenciado de variação em relação ao vidro comum incolor; (c) os vidros refletivos pirolíticos (Figura 3), embora apresentem transmitância muito inferior à do vidro comum incolor, têm essa perda compensada pelo aumento de absortância, o que acarreta similaridade de variação do FS com o vidro incolor;

(d) os vidros refletivos a vácuo Cebrace (Figura 3) diferem dos vidros comuns na variação do FS, tendo redução menos acentuada do FS para os ângulos de $70^{\circ}$ e $80^{\circ}$; e

(e) com os vidros refletivos a vácuo SunGuardian (Figura 3) ocorre o contrário, com a diminuição mais acentuada do FS para os ângulos maiores em relação aos vidros comuns.

As películas comuns (Figura 4) têm o mesmo padrão de variação do FS que o vidro incolor usado como substrato no ensaio delas. 
As películas refletivas (Figura 4) sofrem redução mais acentuada do FS para $70^{\circ}$ e $80^{\circ}$ do que o vidro comum incolor, sendo conveniente a adoção de um material de referência próprio para esse grupo de materiais, mais fiel ao comportamento dele.

Os policarbonatos e o acrílico (Figura 4) têm para o FS o comportamento similar de variação ao dos vidros comuns.

Assim, entre os 60 elementos ensaiados, foram definidos cinco grupos de padrões de variação do FS com o ângulo de incidência:

(a) Grupo1: Vidros comuns, vidros refletivos pirolíticos, películas comuns, policarbonatos e acrílico incolor;

(b) Grupo 2: Vidros laminados comuns;

(c) Grupo 3: Vidros refletivos a vácuo Cebrace;

(d) Grupo 4: Vidros refletivos a vácuo Guardian; e

(e) Grupo 5: Películas refletivas.

Os vidros laminados refletivos vão pertencer sempre ao mesmo grupo que o vidro refletivo utilizado em sua composição, pois têm a mesma variação do FS com o ângulo de incidência que os vidros refletivos originais.

Na Tabela 2 são apresentados os valores médios do FS relativo para cada grupo, que passam a ser os valores do FS do material adotado como referência.
Com base nos valores observados e através de análise de regressão das curvas de variação, foi obtida uma equação genérica (Equação 1), que define a variação do FS dos materiais de referência dos diferentes grupos em função do ângulo de incidência, sendo "A" o ângulo de incidência em radianos, e "b" e "c" os coeficientes das curvas de regressão apresentados na Tabela 3.

$\mathrm{FS}=[\cos (\mathrm{A})]^{\mathrm{b}\left(\mathrm{A}^{\mathrm{c}}\right)}$

Eq. 1

A Figura 6 apresenta a variação do FS dos materiais de referência de cada grupo de materiais em relação ao ângulo de incidência, considerando os valores medidos (a) e os valores calculados pela Equação 1 (b).

\section{Cálculo dos ganhos de calor para um material qualquer}

O cálculo de ganhos de calor ao longo de um período é inicialmente realizado para o material de referência de cada grupo, considerando a radiação solar e o ângulo de incidência dela a cada intervalo de tempo considerado, utilizando-se os valores do fator solar obtidos pela Equação 1. Para obteremse os ganhos para um material qualquer é necessário o conhecimento de a qual grupo ele pertence e do valor do coeficiente de correção, que é o valor do FS para incidência normal do material qualquer.

Tabela 2 - Fator solar (FS) dos materiais de referência dos grupos em função do ângulo

\begin{tabular}{c|c|c|c|c|c}
\hline \multirow{2}{*}{ ÂNGULO } & GRUPO 1 & GRUPO 2 & GRUPO 3 & GRUPO 4 & GRUPO 5 \\
\cline { 2 - 5 } & FS & FS & FS & FS & FS \\
\hline $\mathbf{0}$ & 1,00 & 1,00 & 1,00 & 1,00 & 1,00 \\
\hline $\mathbf{1 0}$ & 1,00 & 1,00 & 1,00 & 1,00 & 1,00 \\
\hline $\mathbf{2 0}$ & 0,99 & 0,99 & 0,99 & 0,98 & 1,00 \\
\hline $\mathbf{3 0}$ & 0,98 & 0,98 & 0,98 & 0.96 & 0,98 \\
\hline $\mathbf{4 0}$ & 0,96 & 0,96 & 0,96 & 0,94 & 0,97 \\
\hline $\mathbf{5 0}$ & 0,93 & 0,92 & 0,93 & 0,88 & 0,95 \\
\hline $\mathbf{6 0}$ & 0,87 & 0,85 & 0,88 & 0,81 & 0,92 \\
\hline $\mathbf{7 0}$ & 0,74 & 0,70 & 0,78 & 0,70 & 0,81 \\
\hline $\mathbf{8 0}$ & 0,47 & 0,40 & 0,55 & 0,39 & 0,67 \\
\hline
\end{tabular}

Tabela 3 - Coeficientes b e c para o cálculo do FS dos materiais de referência

\begin{tabular}{c|c|c}
\hline GRUPOS & b & c \\
\hline GRUPO 1 & 0,243 & 0,186 \\
\hline GRUPO 2 & 0,357 & 0,213 \\
\hline GRUPO 3 & 0,459 & 0,175 \\
\hline GRUPO 4 & 0,549 & 0,287 \\
\hline GRUPO 5 & 0,324 & 0,137 \\
\hline
\end{tabular}




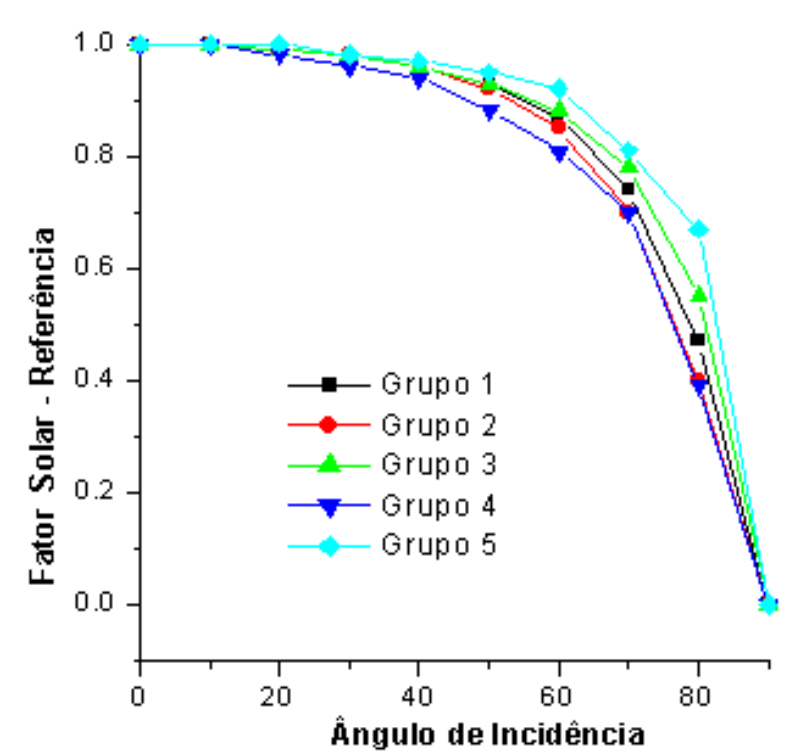

(a) Valores medidos

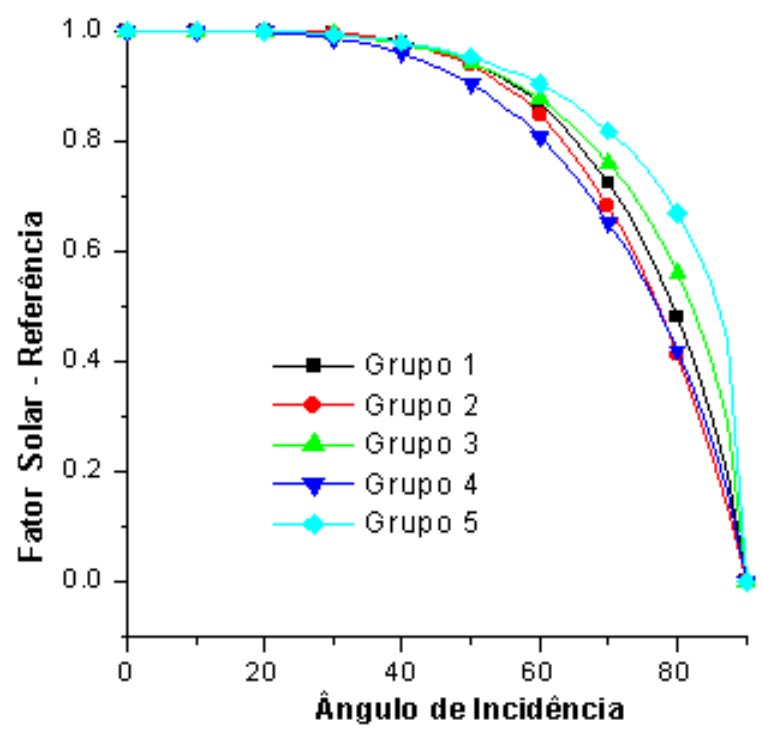

(b) Valores calculados pela Equação 1

Figura 6 - Curvas de variação do FS dos materiais de referência de cada grupo (a) medidos (b) calculados

O número de amostras ensaiadas para obtenção dos dados deste trabalho é reduzido diante do grande número de opções de elementos transparentes existentes no mercado. Essa diversidade, no entanto, está baseada, principalmente, em variações de espessura, em diferentes combinações entre o material-base e a camada de tratamento superficial ou tipo de película aderida, ou, ainda, em composições laminadas e de caixilhos múltiplos, que têm origem, em grande parte, nos elementos ensaiados neste trabalho.

As Tabelas 4 a 8 apresentam os valores obtidos do FS para incidência normal para diferentes materiais transparentes, e o grupo a que os mesmos pertencem. Esses valores são apresentados para velocidades do vento de $2 \mathrm{~m} / \mathrm{s}$ e posição da superfície na vertical.

A Tabela 4 apresenta os valores de FS para incidência normal para os materiais homogêneos, vidros comuns, policarbonatos e acrílico incolor, e a Tabela 5, FSnormal para elementos transparentes com camada superficial metalizada ou película de proteção solar aderida.

A Tabela 6 apresenta os valores para os vidros refletivos ou com película de proteção solar aderida. Dentro desse grupo de elementos, os vidros com tratamento superficial pirolítico (Rp) foram apenas analisados para diferentes espessuras de seus respectivos vidros-base, e os vidros com o tratamento refletivo a vácuo (Rv) ou com as películas aderidas comuns $(\mathrm{Pe})$ ou refletivas (PeR) foram analisados com diferentes espessuras de lâminas de vidro-base incolor.

Na Tabela 7 são apresentados os valores dos vidros laminados incolores ou coloridos compostos de duas lâminas de vidro comum, com o butiral incolor. Nesse caso, em que ambas as lâminas de vidros são de mesma coloração, os valores independem da ordem da espessura das lâminas que o compõem, somente dependendo da espessura total do conjunto do vidro laminado.

No caso dos vidros mistos com lâminas de cores diferentes, eles foram analisados com uma lâmina de vidro colorida e outra incolor, com o butiral incolor. Nesse caso, observou-se que, mantendo-se as respectivas espessuras de cada lâmina, os valores resultantes praticamente independem da ordem das lâminas. A Tabela 7 apresenta os valores para esses vidros.

Na Tabela 8 são apresentados os valores do FS para o ângulo normal para os vidros laminados compostos de duas lâminas de vidro incolor com a película de butiral colorida. Esses valores são específicos para as películas ensaiadas, pois a camada de butiral interior colorida possui um número muito grande de variações, com a combinação das diferentes cores dela, devendo os dados das características ópticas resultantes para as películas em cada caso de combinação ser obtidos por ensaios, ou fornecidos pelo produtor da película. Esses dados devem ser disponibilizados para a película no interior do conjunto do vidro, ou considerando-se dessa maneira. 
Tabela 4 - Fator solar normal (FSnormal) para materiais homogêneos - vidros comuns, policarbonatos e acrílico

\begin{tabular}{|c|c|c|c|c|c|c|c|}
\hline \multirow{3}{*}{ MATERIAL } & \multirow{3}{*}{ COR } & \multirow{3}{*}{ GRUPO } & \multicolumn{5}{|c|}{ ESPESSURA (mm) } \\
\hline & & & 2 & 4 & 6 & 8 & 10 \\
\hline & & & FS & FS & FS & FS & FS \\
\hline \multirow{5}{*}{ VIDROS COMUNS } & Incolor & 1 & 0,88 & 0,86 & 0,84 & 0,83 & 0,81 \\
\hline & Verde & 1 & 0,81 & 0,74 & 0,68 & 0,63 & 0,59 \\
\hline & Bronze & 1 & 0,81 & 0,73 & 0,68 & 0,63 & 0,59 \\
\hline & Cinza & 1 & 0,79 & 0,71 & 0,64 & 0,59 & 0,55 \\
\hline & Azul & 1 & 0,76 & 0,67 & 0,60 & 0,55 & 0,51 \\
\hline \multirow{5}{*}{ POLICARBONATOS } & Incolor & 1 & 0,85 & 0,84 & 0,82 & 0,81 & 0,80 \\
\hline & Verde & 1 & 0,83 & 0,79 & 0,76 & 0,72 & 0,69 \\
\hline & Bronze & 1 & 0,78 & 0,71 & 0,65 & 0,60 & 0,56 \\
\hline & Cinza & 1 & 0,73 & 0,63 & 0,56 & 0,50 & 0,46 \\
\hline & Azul & 1 & 0,83 & 0,80 & 0,77 & 0,74 & 0,71 \\
\hline \multicolumn{2}{|c|}{ ACRÍLICO INCOLOR } & 1 & 0,88 & 0,87 & 0,86 & 0,86 & 0,85 \\
\hline
\end{tabular}

Tabela 5 - Fator solar normal (FSnormal) para elementos transparentes com camada superficial metalizada ou película de proteção solar aderida

\begin{tabular}{|c|c|c|c|c|c|c|}
\hline \multirow{3}{*}{$\begin{array}{c}\text { TIPO } \\
\text { VIDRO } \\
\text { BASE }\end{array}$} & \multirow{3}{*}{$\begin{array}{l}\text { Tratamento } \\
\text { Superficial }\end{array}$} & \multirow{3}{*}{ 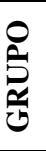 } & \multicolumn{4}{|c|}{$\begin{array}{l}\text { ESPESSURA DO VIDRO BASE } \\
(\mathrm{mm})\end{array}$} \\
\hline & & & 2 & 4 & 6 & 8 \\
\hline & & & FS & FS & FS & FS \\
\hline \multirow{13}{*}{ VIDRO } & Rp Prata & 1 & 0,56 & 0,55 & 0,54 & 0,53 \\
\hline & Rv Prata-neutro & 3 & 0,34 & 0,34 & 0,34 & 0,34 \\
\hline & Rv Prata-médio & 3 & 0,27 & 0,27 & 0,27 & 0,27 \\
\hline & Rv Prata-escuro & 3 & 0,21 & 0,21 & 0,22 & 0,21 \\
\hline & Rv Bronze-neutro & 3 & 0,35 & 0,34 & 0,34 & 0,34 \\
\hline & Rv Bronze-médio & 3 & 0,31 & 0,31 & 0,31 & 0,31 \\
\hline & Rv Bronze-escuro & 3 & 0,22 & 0,22 & 0,22 & 0,22 \\
\hline & Rv Azul-neutro & 3 & 0,49 & 0,48 & 0,48 & 0,47 \\
\hline & Rv Azul-médio & 3 & 0,39 & 0,39 & 0,39 & 0,38 \\
\hline & Rv Azul-escuro & 3 & 0,31 & 0,31 & 0,31 & 0,31 \\
\hline & Rv Azul-intenso & 3 & 0,37 & 0,36 & 0,36 & 0,36 \\
\hline & Rv AzInt-médio & 3 & 0,31 & 0,31 & 0,31 & 0,31 \\
\hline & Rv AzInt-escuro & 3 & 0,25 & 0,25 & 0,25 & 0,25 \\
\hline \multirow[t]{2}{*}{ COMUM } & Pe Fumê G20 & 1 & 0,68 & 0,67 & 0,66 & 0,65 \\
\hline & Pe Fumê G35 & 1 & 0,72 & 0,71 & 0,70 & 0,68 \\
\hline \multirow[t]{12}{*}{ INCOLOR } & Pe Fumê G50 & 1 & 0,76 & 0,74 & 0,73 & 0,72 \\
\hline & Pe Fumê G75 & 1 & 0,82 & 0,80 & 0,79 & 0,77 \\
\hline & Pe Museum & 1 & 0,84 & 0,83 & 0,81 & 0,80 \\
\hline & Pe Violeta & 1 & 0,66 & 0,65 & 0,64 & 0,63 \\
\hline & Pe Bronze & 1 & 0,66 & 0,65 & 0,64 & 0,63 \\
\hline & Pe Verde & 1 & 0,55 & 0,54 & 0,54 & 0,54 \\
\hline & PeR Prata & 5 & 0,32 & 0,33 & 0,33 & 0,33 \\
\hline & PeR Fumê & 5 & 0,27 & 0,28 & 0,28 & 0,28 \\
\hline & PeR Bronze & 5 & 0,27 & 0,27 & 0,28 & 0,28 \\
\hline & PeR Verde & 5 & 0,25 & 0,26 & 0,26 & 0,26 \\
\hline & PeR Ouro & 5 & 0,22 & 0,22 & 0,23 & 0,23 \\
\hline & PeR Azul & 5 & 0,27 & 0,28 & 0,28 & 0,28 \\
\hline VERDE & Rp Verde & 1 & 0,52 & 0,47 & 0,44 & 0,41 \\
\hline BRONZE & Rp Bronze & 1 & 0,56 & 0,51 & 0,47 & 0,44 \\
\hline C. FUMÊ & Rp Fume & 1 & 0,59 & 0,52 & 0,47 & 0,43 \\
\hline
\end{tabular}

Para os vidros laminados refletivos, os dados de refletividade da camada metalizada interior devem ser obtidos de ensaios específicos desses materiais, não sendo possível a adoção dos valores obtidos para o vidro metalizado não laminado, pois, nesse caso, o material que vem após a camada metalizada é o ar $(\eta 2=1)$, e não o outro vidro $(\eta 2=1,62)$, e a mudança no valor do índice de refração desse material altera a refletividade na camada interior. 
Tabela 6 - Fator solar normal (FSnormal) para vidros laminados compostos de duas lâminas de vidro comum de mesma coloração, com película de butiral incolor

\begin{tabular}{|c|c|c|c|c|c|}
\hline \multirow{3}{*}{$\begin{array}{l}\text { COR DO VIDRO } \\
\text { COMUM }\end{array}$} & \multirow{3}{*}{ GRUPO } & \multicolumn{4}{|c|}{$\begin{array}{c}\text { ESPESSURA TOTALVIDRO } \\
\text { COMUM (mm) }\end{array}$} \\
\hline & & 6 & 8 & 10 & 12 \\
\hline & & FS & FS & FS & FS \\
\hline Incolor & 2 & 0,83 & 0,81 & 0,80 & 0,78 \\
\hline Verde & 2 & 0,67 & 0,62 & 0,58 & 0,55 \\
\hline Bronze & 2 & 0,67 & 0,62 & 0,58 & 0,55 \\
\hline Cinza-fumê & 2 & 0,63 & 0,58 & 0,54 & 0,51 \\
\hline Azul & 2 & 0,59 & 0,54 & 0,50 & 0,48 \\
\hline
\end{tabular}

Tabela 7 - Fator solar normal (FSnormal) para vidros laminados compostos de uma lâmina de vidro comum incolor e outra de vidro colorido, com película de butiral incolor

\begin{tabular}{|c|c|c|c|c|c|}
\hline \multirow{3}{*}{$\begin{array}{c}\text { VIDRO } \\
\text { COLORIDO }\end{array}$} & \multirow{3}{*}{ GRUPO } & \multirow{3}{*}{$\begin{array}{l}\text { ESPESSURA VIDRO } \\
\text { COLORIDO (mm) }\end{array}$} & \multicolumn{3}{|c|}{$\begin{array}{l}\text { ESPESSURA VIDRO COMUM } \\
\text { INCOLOR (mm) }\end{array}$} \\
\hline & & & 4 & 6 & 8 \\
\hline & & & FS & FS & FS \\
\hline \multirow{3}{*}{ Verde } & \multirow{3}{*}{2} & 3 & $\overline{0,74}$ & & \\
\hline & & 4 & 0,70 & 0,69 & \\
\hline & & 6 & 0,65 & 0,64 & 0,63 \\
\hline \multirow{3}{*}{ Bronze } & \multirow{3}{*}{2} & 3 & 0,74 & & \\
\hline & & 4 & 0,71 & 0,70 & \\
\hline & & 6 & 0,66 & 0,65 & 0,64 \\
\hline \multirow{3}{*}{ Cinza-fumê } & \multirow{3}{*}{2} & 3 & 0,71 & & \\
\hline & & 4 & 0,68 & 0,67 & \\
\hline & & 6 & 0,62 & 0,61 & 0,60 \\
\hline \multirow{3}{*}{ Azul } & \multirow{3}{*}{2} & 3 & 0,69 & & \\
\hline & & 4 & 0,64 & 0,63 & \\
\hline & & 6 & 0,58 & 0,57 & 0,57 \\
\hline
\end{tabular}

Tabela 8 - Fator solar normal (FSnormal) para vidros laminados compostos de duas lâminas de vidro comum incolor, com película de butiral colorida

\begin{tabular}{|c|c|c|c|c|c|}
\hline \multirow{3}{*}{$\begin{array}{c}\text { COR DA } \\
\text { PELÍCULA }\end{array}$} & \multirow{3}{*}{ GRUPO } & \multicolumn{4}{|c|}{ ESPESSURA TOTAL (mm) } \\
\hline & & 6 & 8 & 10 & 12 \\
\hline & & FS & FS & FS & FS \\
\hline Verde & 2 & 0,76 & 0,74 & 0,73 & 0,72 \\
\hline Bronze-claro & 2 & 0,70 & 0,69 & 0,67 & 0,66 \\
\hline Bronze-escuro & 2 & 0,59 & 0,58 & 0,58 & 0,57 \\
\hline Fume & 2 & 0,66 & 0,65 & 0,64 & 0,64 \\
\hline Azul-claro & 2 & 0,80 & 0,79 & 0,78 & 0,76 \\
\hline Rosa & 2 & 0,70 & 0,69 & 0,68 & 0,67 \\
\hline Azul-escuro & 2 & 0,71 & 0,70 & 0,69 & 0,68 \\
\hline
\end{tabular}

Em todas as composições de vidros laminados estudadas é considerada uma limitação de fabricação em que a relação entre a maior e a menor espessura não deve ultrapassar 1,5. Essa limitação é para evitar quebras que ocorrem na autoclavagem, processo pelo qual passam os vidros laminados para adquirir suas características finais.

Para os materiais que não constam dessas tabelas, podem ser adotados os valores de FS apresentados nos catálogos dos fabricantes para incidência normal.

\section{Conclusões}

O método apresentado neste trabalho tem por objetivo facilitar a consideração do ângulo de incidência da radiação solar no cálculo de ganhos de calor através de elementos transparentes. Para isso, foram criados grupos de materiais transparentes que apresentam curvas similares de 
variação do fator solar em relação ao ângulo de incidência solar. A média para cada ângulo dos valores dessas curvas define os valores de FS para um material hipotético de referência de cada grupo, definindo a curva de variação desse material.

Uma equação genérica de variação do FS foi obtida por análise de regressão dessas curvas, tendo parâmetros específicos para cada grupo. Essa equação possibilita o cálculo do FS para qualquer ângulo de incidência de 0 a 90 graus, para todos os materiais de referência. Os valores do FS para determinado material podem, então, ser obtidos para qualquer ângulo, pela multiplicação do valor de seu FS para incidência normal (FSnormal) pelo valor do FS, para o ângulo desejado, do material de referência do grupo a que ele pertence.

Para o cálculo dos ganhos de calor solar ao longo de um período são utilizados os valores obtidos para o material de referência de determinado grupo, resultando um valor de ganho solar de referência. Este valor é corrigido posteriormente, para obter-se o ganho para qualquer material do grupo, a partir da utilização de um coeficiente, que no método apresentado neste trabalho é o FS para a incidência normal (FSnormal) desse material.

A precisão do cálculo proposto está associada à semelhança entre os comportamentos dos materiais de cada grupo, quanto à influência exercida pelo ângulo de incidência sobre o FS, pois, para cada material, um único valor do coeficiente de correção deve ser válido para qualquer ângulo.

A definição dos grupos e das curvas de referência de variação do FS com o ângulo de incidência simplifica também a necessidade de ensaios necessários à caracterização de determinado elemento transparente, que esteja enquadrado dentro dos grupos estabelecidos. Dessa forma, poderão ser obtidos os valores do FS a ângulos variados para um grande número de opções de espessuras e composições de materiais transparentes, bastando ter conhecimento de a qual grupo o material pertence e calculando-se, ou obtendo-se por ensaio, o valor do FS para a incidência normal. Isso facilita o cálculo dos ganhos de carga térmica solar para qualquer situação de incidência da radiação.

O fator solar para incidência normal é dado de entrada desse método. Caso não estiver disponível nas tabelas apresentadas neste trabalho, pode ser obtido na literatura especializada ou mesmo nos catálogos dos fabricantes. A definição do grupo a que pertence um material diferente dos citados neste trabalho pode ser feita a partir da comparação de seu processo de fabricação e de sua composição com os materiais aqui apresentados.

\section{Referências} AMERICAN SOCIETY OF HEATING,
REFRIGERATING AND AIR CONDITIONING
ENGINEERS. Fundamentals. Atlanta, 1997. cap. 29.

AMERICAN SOCIETY FOR TESTING AND

MATERIALS. E892-87: standard tables for terrestrial solar spectral irradiance at air mass 1.5 for a $37^{\circ}$ tilted surface. West Conshohocken, 1987.

CARAM, R. M. Caracterização Ótica de Materiais Transparentes e Sua Relação Com o Conforto Ambiental em Edificações. $165 \mathrm{f}$. Campinas, 1998. Tese (Doutorado em Engenharia Civil) - Faculdade de Engenharia Civil, Universidade de Campinas, Campinas, 1998.

CLEDWYN-DAVIES, D. N. Float in Glass Architecture. Glass Technology, v. 34, n. 6, p. 223-228, 1993.

COMPANHIA VIDRARIA SANTA MARINA. O Vidro na Arquitetura. São Paulo: Projeto Editores Associados, 1993.

MARINOSKI, D. L. Desenvolvimento de Um Calorímetro Para Determinação do Fator Solar de Vidros e Janelas. 298 f. Florianópolis, 2010. Tese (Doutorado em Engenharia Civil) Departamento de Engenharia Civil, Universidade Federal de Santa Catarina, Florianópolis, 2010.

PFROMMER, P. et al. The Radiation Transfer Through Coated and Tinted Glazing. Solar Energy, v. 54, n. 5, p. 287-299, 1995.

SANTOS, J. C. P. Avaliação do Desempenho Térmico e Visual de Elementos Transparentes Frente à Radiação Solar. 2002. Tese (Doutorado em Ciência e Engenharia de Materiais) Departamento de Ciência e Engenharia de Materiais, Universidade de São Paulo, São Carlos, 2002.

SANTOS, J. C. P.; FERNANDES, M. R. Comportamento Ótico de Vidros Translúcidos Frente à Radiação Solar. In: ENCONTRO

NACIONAL DE TECNOLOGIA NO AMBIENTE CONSTRUÍDO, 12., Fortaleza, 2008. Anais... Fortaleza: ANTAC, 2008.

SANTOS, J. C. P.; FERNANDES, M. R. Comportamento Ótico de Policarbonatos Translúcidos Frente à Radiação Solar. In: ENCONTRO NACIONAL DE CONFORTO NO AMBIENTE CONSTRUÍDO, 10., Natal, 2009. Anais... Natal: ANTAC, 2009. 
SANTOS, J. C. P.; RORIZ, M. Influência do Ângulo de Incidência na Transmitância de Luz Natural Através de Materiais Transparentes.

Ambiente Construído, Porto Alegre, v. 7, n. 4, p. 123-135, out./dez. 2007.

Revista Ambiente Construído

Associação Nacional de Tecnologia do Ambiente Construído

Av. Osvaldo Aranha, 99 - 3o andar, Centro

Porto Alegre - RS - Brasil

CEP 90035-190

Telefone: +55 (51) 3308-4084

Fax: +55 (51) 3308-4054

www. seer. ufrgs. br/ ambienteconstruido

E-mail: ambienteconstruido@ufrgs.br 